\title{
Ranking the Leading Journals in Finance and Accounting Based on Quantity and Quality Citations
}

\author{
Michael McAleer \\ Department of Quantitative Finance \\ National Tsing Hua University \\ Taiwan \\ and \\ Econometric Institute \\ Erasmus School of Economics \\ Erasmus University Rotterdam \\ and \\ Tinbergen Institute \\ The Netherlands \\ and \\ Department of Quantitative Economics \\ Complutense University of Madrid
}




\section{Introduction - 1}

The perceived quality and impact of academic journals are important in evaluating the perceived research performance of individual researchers for hiring, tenure and promotion decisions.

Rightly or wrongly, such perceived journal quality and impact are typically used as a proxy for the quality of an academic paper, as publication in a highly regarded journal is seen as imparting an important signal regarding the purported quality of the published paper. 


\section{Introduction - 2}

The determination of the leading journals in any discipline can be based on a wide range of quantitative and qualitative assessments, ranging from expert (and possibly subjective) assessments of journal impact, evaluation of high quality and high impact articles, and the use of quantifiable bibliometric Research Assessment Measures (RAMs). 


\section{Introduction - 3}

The leading database for generating RAMs to evaluate the quality and impact of academic journals, as well as the research performance of individual researchers, is the Thomson Reuters ISI Web of Science database.

Virtually all RAMs are based on alternative transformations of citations data. 


\section{Introduction - 4}

As the alternative RAMs that are provided in Thomson Reuters ISI and in several recent publications may not be widely known, this section provides a brief description and definition of 16 RAMs that may be calculated annually or updated daily. 


\section{Annual RAM}

With four exceptions, namely Eigenfactor, Article Influence (AI), Cited Article Influence (CAI) and Index of Citations Quality (ICQ), all existing RAMs are based on citations data and are reported separately for the sciences and social sciences. RAMs may be computed annually or updated daily. The annual RAMs given below are calculated for a Journal Citations Reports (JCR) calendar year, which is the year before the annual RAMs are released. For example, the most recent RAMs were released by ISI (2013) in late-June 2013 for the JCR calendar year 2012. 


\section{(1) 2-year impact factor including journal self citations (2YIF)}

The classic 2-year impact factor including journal self citations (2YIF) of a journal is typically referred to as "the impact factor", is calculated annually, and is defined as "Total citations in a year to papers published in a journal in the previous 2 years / Total papers published in a journal in the previous 2 years”. The choice of 2 years by ISI is arbitrary. It is widely held in the academic community, and certainly by the editors and publishers of journals, that a higher 2YIF is better than lower. 


\section{(2) 2-year impact factor excluding journal self citations (2YIF*)}

ISI also reports a 2-year impact factor without journal self citations (that is, citations to a journal in which a citing paper is published), which is calculated annually. As this impact factor is not widely known or used, Chang et al. (2011c) refer to this RAM as 2YIF*. Although 2YIF* is rarely reported, a higher value would be preferred to lower. 


\section{(3) 5-year impact factor including journal self citations (5YIF)}

The 5-year impact factor including journal self citations (5YIF) of a journal is calculated annually, and is defined as "Total citations in a year to papers published in a journal in the previous 5 years / Total papers published in a journal in the previous 5 years.” The choice of 5 years by ISI is arbitrary. Although 5YIF is not widely reported, a higher value would be preferred to lower. 


\section{(4) Immediacy, or 0-year impact factor incl. journal self citations (OYIF)}

Immediacy is a zero-year impact factor including journal self citations (OYIF) of a journal, is calculated annually, and is defined as "Total citations to papers published in a journal in the same year / Total papers published in a journal in the same year.” The choice of the same year by ISI is arbitrary, but the nature of Immediacy makes it clear that a very short run outcome is under consideration. Although Immediacy is not frequently reported, a higher value would be preferred to lower. 
(5) 5YIF Divided by 2YIF (5YD2)

As both 2YIF and 5YIF include journal self citations, if it is assumed that journal self citations are uniformly distributed over the 5-year period for calculating 5YIF, their ratio will eliminate the effect of journal self citations and capture the increase in the citation rate over time. In any event, the impact of journal self citations should be mitigated with the ratio of 5YIF to 2YIF. We define a new dynamic RAM as 5YD2 as "5YD2 = 5YIF / 2YIF". In the sciences, where citations are observed with a frequency of weeks and months rather than years, it is typically the case that 5YIF $<2$ YIF, whereas the reverse, 5YIF > 2YIF, seems to hold generally in the social sciences. 


\section{(6) Eigenfactor (or Journal Influence)}

The Eigenfactor score (see Bergstrom (2007), Bergstrom and West (2008), Bergstrom, West and Wiseman (2008)) is calculated annually, and is defined as: "The Eigenfactor Score calculation is based on the number of times articles from the journal published in the past five years have been cited in the JCR year, but it also considers which journals have contributed these citations so that highly cited journals will influence the network more than lesser cited journals. References from one article in a journal to another article from the same journal are removed, so that Eigenfactor Scores are not influenced by journal self-citation.” 
The value of the threshold that separates 'highly cited' from 'lesser cited' journals, as well as how the former might 'influence the network more' than the latter, are based on the Eigenfactor score of the citing journal. Thus, Eigenfactor might usefully be interpreted as a weighted total citations score, or a "Journal Influence" measure. A higher Eigenfactor score would be preferred to lower. 


\section{(7) Article Influence (or Journal Influence per Article)}

Article Influence (see Bergstrom (2007), Bergstrom and West (2008), Bergstrom, West and Wiseman (2008)) measures the relative importance of a journal's citation influence on a per-article basis. Despite the misleading suggestion of measuring "Article Influence”, as each journal has only a single "Article Influence" score, this RAM is actually a "Journal Influence per Article" score. Article Influence is a scaled Eigenfactor score, is calculated annually, is standardized to have a mean of one across all journals in the Thomson Reuters ISI database, and is defined as "Eigenfactor score divided by the fraction of all articles published by a journal.” A higher Article Influence would be preferred to lower. 


\section{(8) IFI}

The ratio of 2YIF to 2YIF* is intended to capture how journal self citations can inflate the impact factor of a journal, whether this is an unconscious self-promotion decision made independently by publishing authors or as an administrative decision undertaken by a journal's editors and/or publishers. Chang et al. (2011a) define Impact Factor Inflation (IFI) as "IFI = 2YIF / 2YIF*". The minimum value for IFI is 1 , with any value above the minimum capturing the effect of journal self citations on the 2-year impact factor. A lower IFI would be preferred to higher. 


\section{(9) H-STAR}

ISI has implicitly recognized the inflation in journal self citations by calculating an impact factor that excludes self citations, and provides data on journal self citations, both historically (for the life of the journal) and for the preceding two years, in calculating 2YIF. Chang et al. (2011b) define the Self-citation Threshold Approval Rating (STAR) as the percentage difference between citations in other journals and journal self citations. If HS = historical journal self citations, then Historical STAR (H-STAR) is defined as "H-STAR $=[(100-H S)-\mathrm{HS}]=(100-2 \mathrm{HS})$ ". If $\mathrm{HS}=0$ (minimum), 50 or 100 (maximum) percent, for example, H-STAR $=100,0$ and -100 , respectively. A higher H-STAR would be preferred to lower. 


\section{(10) 2Y-STAR}

H-STAR examines the self-citation threshold approval rating over the historical period for which data for a journal are available, namely from the inclusion of the journal in ISI, whereas 2Y-STAR examines the self-citation threshold approval rating based on data for the preceding two years. If 2YS = journal self citations over the preceding 2-year period, then the 2-Year STAR is defined by Chang et al. (2011b) as "2Y-STAR = [(100-2YS) $2 \mathrm{YS}]=(100-2(2 \mathrm{YS})) "$. If $2 \mathrm{YS}=0$ (minimum), 50 or 100 (maximum) percent, for example, 2Y-STAR $=100,0$ and -100 , respectively. A higher 2Y-STAR would be preferred to lower. 


\section{(11) Escalating Self Citations (ESC)}

As self citations for many journals in the sciences and social sciences have been increasing over time, it would seem useful to present a dynamic RAM that captures such an escalation over time. The difference given by 2 YS HS measures Escalating Self Citations in journals over the most recent 2 years relative to the historical period for calculating citations, which will differ across journals. We define a new dynamic RAM as "ESC = 2YS $\mathrm{HS}=(\mathrm{H}-\mathrm{STAR}-2 \mathrm{Y}-\mathrm{STAR}) / 2$ ". Given the range of each of H-STAR and 2Y-STAR is $(-100,100)$, the range of ESC is also $(-100,100)$, with -100 denoting minimum, and 100 denoting maximum, escalation. A lower ESC would be preferred to higher. 


\section{(12) Index of Citations Quality (ICQ)}

Wilhite and Fong (2012) and Chang, et al. (2013), among others, have argued the well-known practice of coercive journal citations by both editors and publishers distorts the intended meaning and interpretation of journal impact and influence. Chang and McAleer (2014b, c) suggested the following Index of Citations Quality (ICQ) to try to evaluate the impact of coercive self citations: "ICQ = AI / 5YIF = Quality Weighted Citations / Total Citations $=$ "Quality weighted citations in the past 5 years, excluding journal self citations" / "Total citations in the previous 5 years, including journal self citations”. A higher ICQ would generally be preferred to lower. 


\section{Daily Updated RAM}

Some RAMs are updated daily, and are reported for a given day in a calendar year rather than for a JCR year. 


\section{(13) C3PO}

ISI reports the mean number of citations for a journal, namely total citations up to a given day divided by the number of papers published in a journal up to the same day, as the "average" number of citations. In order to distinguish the mean from the median and mode, the C3PO of an ISI journal on any given day is defined by Chang et al. (2011a) as "C3PO (Citation Performance Per Paper Online) $=$ Total citations to a journal $/$ Total papers published in a journal.” A higher CЗPO would be preferred to lower. [Note: C3PO should not be confused with C-3PO, the Star Wars android.] 


\section{(14) h-index}

The h-index (Hirsch, 2005)) was originally proposed to assess the scientific research productivity and citations impact of individual researchers. However, the h-index can also be calculated for journals, and should be interpreted as assessing the impact or influence of highly cited journal publications. The h-index of a journal on any given day is based on historically cited and citing papers, including journal self citations, and is defined as "h-index = number of published papers, where each has at least $\mathrm{h}$ citations." The h-index differs from an impact factor in that the h-index measures the number of highly cited papers historically. A higher h-index would be preferred to lower. 


\section{(15) PI-BETA}

This RAM measures the proportion of papers in a journal that has never been cited, As such, PI-BETA is, in effect, a rejection rate of a journal after publication. Chang et al. (2011c) argue that lack of citations of a published paper, especially if it is not a recent publication, reflects on the quality of a journal by exposing: (i) what might be considered as incorrect decisions by the members of the editorial board of a journal; and (ii) the lost opportunities of papers that might have been cited had they not been rejected by the journal. 
Chang et al. (2011c) propose that a paper with zero citations in ISI journals can be measured by PI-BETA (= Papers Ignored (PI) - By Even The Authors (BETA)), which is calculated for an ISI journal on any given day as "Number of papers with zero citations in a journal / Total papers published in a journal.” As journals would typically prefer a higher proportion of published papers being cited rather than ignored, a lower PIBETA would be preferred to higher. 


\section{(16) CAI}

Article Influence is intended to measure the average influence of an article across the sciences and social sciences. As an article with zero citations typically does not have any (academic) influence, a more suitable measure of the influence of cited articles would seem to be Cited Article Influence (CAI). Chang et al. (2011b) define CAI as “CAI = (1 - PI-BETA)(Article Influence)”. If PI-BETA = 0, then CAI is equivalent to Article Influence; if PI-BETA $=1$, then CAI $=0$. As Article Influence is calculated annually and PI-BETA is updated daily, CAI may be updated daily. A higher CAI would be preferred to lower. 


\section{Summary - 1}

The journals rankings presented above indicate clearly that there are three leading journals in Finance, namely Journal of Finance, Journal of Financial Economics and Review of Financial Studies.

These journals form an exclusive club in terms of the RAMs that measure journal quality and impact based on alternative measures of journal citations. 


\section{Summary - 2}

The next two journals in terms of overall quality and impact are Journal of Accounting and Economics and Journal of Monetary Economics.

The Journal of Financial and Quantitative Analysis is not in the top 10 journals in Finance, despite a widely-held perception in the discipline that it might be just outside the top 3 journals. 


\section{Summary - 3}

As Accounting does not have a separate classification in ISI, the top 3 journals in the sub-category of Accounting in the ISI category of "Business - Finance", are Journal of Accounting and Economics, Accounting Review, and Journal of Accounting Research. 
Table 1

16 Research Assessment Measures (RAM) for 89 Leading Finance Journals

\begin{tabular}{|c|c|c|c|c|c|c|c|c|c|c|c|c|c|c|c|c|}
\hline Journal & 2YIF & 2YIF* & IFI & 5YIF & $\begin{array}{l}\text { Imme- } \\
\text { diacy }\end{array}$ & 5YD2 & $\begin{array}{c}\text { h- } \\
\text { index }\end{array}$ & СЗРО & $\begin{array}{l}\text { PI- } \\
\text { BETA }\end{array}$ & $\begin{array}{l}\text { Eigen- } \\
\text { factor }\end{array}$ & AI & CAI & ICQ & $\begin{array}{c}\text { H- } \\
\text { STAR }\end{array}$ & $\begin{array}{l}\text { 2Y- } \\
\text { STAR }\end{array}$ & ESC \\
\hline J FINANC & 4.333 & 3.984 & 1.088 & 6.185 & 0.867 & 1.427 & 204 & 31.11 & 0.354 & 0.05733 & 8.824 & 5.7 & 1.427 & 96 & 94 & 1 \\
\hline J ACCOUNT ECON & 3.912 & 3.294 & 1.188 & 4.023 & 0.289 & 1.028 & 96 & 42.7 & 0.085 & 0.00741 & 2.453 & 2.244 & 0.61 & 82 & 70 & 6 \\
\hline J FINANC ECON & 3.424 & 3.038 & 1.127 & 5.087 & 0.669 & 1.486 & 179 & 72.57 & 0.082 & 0.05835 & 6.327 & 5.808 & 1.244 & 88 & 78 & 5 \\
\hline REV FINANC STUD & 3.256 & 2.863 & 1.137 & 5.367 & 0.421 & 1.648 & 109 & 37.75 & 0.108 & 0.06476 & 7.242 & 6.46 & 1.349 & 88 & 76 & 6 \\
\hline IMF ECON REV & 2.529 & 2.206 & 1.146 & 2.559 & 0 & 1.012 & 11 & 3.72 & 0.419 & 0.00248 & 4.172 & 2.424 & 1.63 & 74 & 76 & -1 \\
\hline ACCOUNT REV & 2.319 & 1.743 & 1.33 & 3.204 & 0.603 & 1.382 & 82 & 6.25 & 0.568 & 0.00795 & 1.474 & 0.637 & 0.46 & 74 & 52 & 11 \\
\hline IMF STAFF PAPERS & 2.312 & 2.312 & 1 & 1.344 & 0 & 0.581 & 29 & 9.24 & 0.147 & 0.00244 & 1.454 & 1.24 & 1.082 & 100 & 100 & 0 \\
\hline J FINANC INTERMED & 2.208 & 2.132 & 1.036 & 2.46 & 0.107 & 1.114 & 40 & 14.37 & 0.204 & 0.00582 & 2.688 & 2.14 & 1.093 & 94 & 94 & 0 \\
\hline J ACCOUNT RES & 2.192 & 1.863 & 1.177 & 3.368 & 0.256 & 1.536 & 88 & 20.85 & 0.196 & 0.00703 & 2.21 & 1.777 & 0.656 & 90 & 70 & 10 \\
\hline ACCOUNT ORG SOC & 1.867 & 1.44 & 1.297 & 3.143 & 0.125 & 1.683 & 68 & 20.81 & 0.095 & 0.00364 & 1.028 & 0.93 & 0.327 & 76 & 56 & 10 \\
\hline $\begin{array}{l}\text { J RISK } \\
\text { UNCERTAINTY }\end{array}$ & 1.771 & 1.25 & 1.417 & 2.016 & 0.167 & 1.138 & 53 & 25.16 & 0.109 & 0.0036 & 1.625 & 1.448 & 0.806 & 86 & 58 & 14 \\
\hline J MONETARY ECON & 1.649 & 1.491 & 1.106 & 2.529 & 0.163 & 1.534 & 115 & 30.83 & 0.164 & 0.02718 & 3.742 & 3.128 & 1.48 & 96 & 82 & 7 \\
\hline $\begin{array}{l}\text { J FINANC QUANT } \\
\text { ANAL }\end{array}$ & 1.636 & 1.579 & 1.036 & 2.13 & 0.214 & 1.302 & 79 & 15.39 & 0.206 & 0.01169 & 2.645 & 2.1 & 1.242 & 98 & 94 & 2 \\
\hline $\begin{array}{l}\text { CONTEMP ACCOUNT } \\
\text { RES }\end{array}$ & 1.564 & 1.385 & 1.129 & 2.154 & 0.186 & 1.377 & 31 & 8.79 & 0.28 & 0.00348 & 1.094 & 0.788 & 0.508 & 80 & 78 & 1 \\
\hline J FINANC STABIL & 1.463 & 0.878 & 1.666 & 1.568 & 0.208 & 1.072 & 13 & 3.33 & 0.386 & 0.00095 & - & - & - & 42 & 20 & 11 \\
\hline REV FINANC & 1.44 & 1.4 & 1.029 & 2.456 & 0.357 & 1.706 & 17 & 4.41 & 0.372 & 0.00477 & - & - & - & 96 & 96 & 0 \\
\hline CORP GOV-OXFORD & 1.4 & 0.431 & 3.248 & 1.581 & 0.156 & 1.129 & 11 & 2.77 & 0.388 & 0.00164 & 0.364 & 0.223 & 0.23 & 42 & -38 & 40 \\
\hline $\begin{array}{l}\text { MANAGE ACCOUNT } \\
\text { RES }\end{array}$ & 1.366 & 0.878 & 1.556 & 2.531 & 0.118 & 1.853 & 11 & 4.4 & 0.317 & 0.00105 & - & - & - & 68 & 30 & 19 \\
\hline REV ACCOUNT STUD & 1.364 & 1.2 & 1.137 & 1.899 & 0.219 & 1.392 & 27 & 8.45 & 0.304 & 0.00231 & 1.214 & 0.845 & 0.639 & 82 & 76 & 3 \\
\hline FINANC MANAGE & 1.33 & 0.907 & 1.466 & 1.568 & 0.235 & 1.179 & 46 & 8.52 & 0.219 & 0.00345 & 1.069 & 0.835 & 0.682 & 80 & 38 & 21 \\
\hline
\end{tabular}




\begin{tabular}{|c|c|c|c|c|c|c|c|c|c|c|c|c|c|c|c|c|}
\hline Journal & 2YIF & 2YIF* & IFI & 5YIF & Immediacy & 5YD2 & $\begin{array}{c}\mathbf{h}- \\
\text { index }\end{array}$ & СЗРО & $\begin{array}{c}\text { PI- } \\
\text { BETA }\end{array}$ & Eigenfactor & $\begin{array}{c}\text { Article } \\
\text { Influence }\end{array}$ & CAI & ICQ & $\begin{array}{c}\text { H- } \\
\text { STAR } \\
\end{array}$ & $\begin{array}{c}\text { 2Y- } \\
\text { STAR }\end{array}$ & ESC \\
\hline J OPER RISK & 0.182 & 0.182 & 1 & 0.427 & 0 & 2.346 & 11 & 3.2 & 0.466 & 0.00032 & - & - & - & 100 & 100 & 0 \\
\hline INVEST ANAL J & 0.176 & 0.059 & 2.983 & 0.313 & 0.182 & 1.778 & 3 & 0.69 & 0.662 & 0.00003 & 0.034 & 0.011 & 0.109 & -14 & -32 & 9 \\
\hline REV DERIV RES & 0.16 & 0.16 & 1 & 0.326 & 0 & 2.038 & 4 & 0.96 & 0.563 & 0.00021 & - & - & - & 100 & 100 & 0 \\
\hline J RISK & 0.152 & 0.152 & 1 & 0.507 & 0 & 3.336 & 5 & 0.96 & 0.632 & 0.00037 & - & - & - & 100 & 100 & 0 \\
\hline INT INSOLV REV & 0.15 & 0.1 & 1.5 & 0.258 & 0 & 1.72 & 2 & 0.36 & 0.709 & 0.00006 & - & - & - & 8 & 34 & -13 \\
\hline $\begin{array}{l}\text { REV ESP FINANC } \\
\text { CONTA }\end{array}$ & 0.106 & 0.043 & 2.465 & 0.167 & 0.048 & 1.575 & 2 & 0.15 & 0.876 & 0.00004 & - & - & - & -8 & -20 & 6 \\
\hline J CREDIT RISK & 0.091 & 0.091 & 1 & 0.389 & 0 & 4.275 & 4 & 0.68 & 0.633 & 0.0003 & - & - & - & 100 & 100 & 0 \\
\hline $\begin{array}{l}\text { J RISK MODEL } \\
\text { VALIDAT }\end{array}$ & 0.031 & 0.031 & 1 & 0.146 & 0 & 4.71 & 3 & 0.6 & 0.67 & 0.0001 & - & - & - & 100 & 100 & 0 \\
\hline JASSA & 0 & 0 & - & 0.036 & 0 & - & 2 & 0.07 & 0.95 & 0.00001 & - & - & - & - & - & - \\
\hline Mean & 1.006 & 0.806 & 1.679 & 1.424 & 0.171 & 1.577 & 29 & 7.406 & 0.396 & 0.00454 & 1.363 & 1.025 & 0.719 & 71 & 57 & 7 \\
\hline Low & 0 & 0 & 1 & 0.036 & 0 & 0.49 & 2 & 0.02 & 0.082 & 0.00001 & 0.034 & 0.001 & 0.109 & -64 & -92 & -13 \\
\hline High & 4.333 & 3.984 & 27.8 & 6.185 & 0.867 & 4.71 & 204 & 72.57 & 0.982 & 0.06476 & 8.824 & 6.46 & 1.772 & 100 & 100 & 46 \\
\hline
\end{tabular}

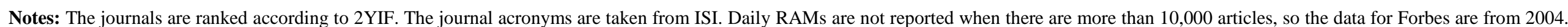
Data for all other journals are from the year of their inclusion in ISI. The data were downloaded from ISI on 14 May 2014. 
Table 2

Correlations of 16 RAM for 58 Leading Finance Journals

\begin{tabular}{|c|c|c|c|c|c|c|c|c|c|c|c|c|c|c|c|c|}
\hline Journal & 2 YIF & 2YIF* & IFI & $5 Y I F$ & Immediacy & 5YD2 & h-index & СЗРО & PI-BETA & Eigenfactor & $\begin{array}{c}\text { Article } \\
\text { Influence }\end{array}$ & CAI & ICQ & H-STAR & 2Y-STAR & ESC \\
\hline 2YIF & 1 & & & & & & & & & & & & & & & \\
\hline 2YIF* & 0.974 & 1 & & & & & & & & & & & & & & \\
\hline IFI & -0.217 & -0.383 & 1 & & & & & & & & & & & & & \\
\hline 5YIF & 0.936 & 0.917 & -0.244 & 1 & & & & & & & & & & & & \\
\hline Immediacy & 0.615 & 0.583 & -0.062 & 0.685 & 1 & & & & & & & & & & & \\
\hline 5YD2 & -0.054 & -0.029 & -0.099 & 0.222 & 0.069 & 1 & & & & & & & & & & \\
\hline h-index & 0.801 & 0.795 & -0.234 & 0.873 & 0.769 & 0.173 & 1 & & & & & & & & & \\
\hline CЗPO & 0.774 & 0.772 & -0.245 & 0.822 & 0.597 & 0.165 & 0.852 & 1 & & & & & & & & \\
\hline PI-BETA & -0.458 & -0.45 & 0.224 & -0.464 & -0.187 & -0.205 & -0.455 & -0.585 & 1 & & & & & & & \\
\hline Eigenfactor & 0.71 & 0.719 & -0.179 & 0.816 & 0.698 & 0.158 & 0.844 & 0.775 & -0.3 & 1 & & & & & & \\
\hline $\begin{array}{l}\text { Article } \\
\text { Influence }\end{array}$ & 0.846 & 0.876 & -0.3 & 0.896 & 0.653 & 0.12 & 0.828 & 0.762 & -0.361 & 0.901 & 1 & & & & & \\
\hline CAI & 0.835 & 0.862 & -0.291 & 0.893 & 0.629 & 0.134 & 0.835 & 0.845 & -0.446 & 0.927 & 0.979 & 1 & & & & \\
\hline ICQ & 0.452 & 0.548 & -0.524 & 0.422 & 0.264 & -0.083 & 0.424 & 0.42 & -0.296 & 0.472 & 0.674 & 0.645 & 1 & & & \\
\hline H-STAR & 0.326 & 0.427 & -0.715 & 0.328 & 0.161 & 0.025 & 0.335 & 0.327 & -0.275 & 0.247 & 0.373 & 0.366 & 0.633 & 1 & & \\
\hline 2Y-STAR & 0.246 & 0.422 & -0.946 & 0.259 & 0.052 & 0.064 & 0.231 & 0.253 & -0.207 & 0.202 & 0.342 & 0.329 & 0.584 & 0.776 & 1 & \\
\hline ESC & -0.054 & -0.226 & 0.750 & -0.072 & 0.081 & -0.074 & -0.021 & -0.065 & 0.043 & -0.065 & -0.155 & -0.142 & -0.27 & -0.198 & -0.772 & 1 \\
\hline
\end{tabular}


Table 3

Correlations of 13 RAM for 88 Leading Finance Journals

\begin{tabular}{|c|c|c|c|c|c|c|c|c|c|c|c|c|c|}
\hline Journal & 2YIF & 2YIF* & IFI & 5YIF & Immediacy & 5YD2 & h-index & СЗРО & PI-BETA & Eigenfactor & H-STAR & 2Y-STAR & ESC \\
\hline 2YIF & 1 & & & & & & & & & & & & \\
\hline 2 YIF* $^{*}$ & 0.971 & 1 & & & & & & & & & & & \\
\hline IFI & -0.119 & -0.168 & 1 & & & & & & & & & & \\
\hline $5 Y I F$ & 0.905 & 0.885 & -0.125 & 1 & & & & & & & & & \\
\hline Immediacy & 0.603 & 0.546 & 0.049 & 0.642 & 1 & & & & & & & & \\
\hline 5YD2 & -0.284 & -0.24 & -0.045 & 0.003 & -0.183 & 1 & & & & & & & \\
\hline h-index & 0.804 & 0.809 & -0.106 & 0.8 & 0.632 & -0.154 & 1 & & & & & & \\
\hline СЗРО & 0.779 & 0.788 & -0.102 & 0.765 & 0.509 & -0.127 & 0.874 & 1 & & & & & \\
\hline PI-BETA & -0.585 & -0.563 & 0.192 & -0.525 & -0.291 & 0.261 & -0.543 & -0.612 & 1 & & & & \\
\hline Eigenfactor & 0.7 & 0.72 & -0.07 & 0.755 & 0.575 & -0.055 & 0.842 & 0.786 & -0.345 & 1 & & & \\
\hline H-STAR & 0.241 & 0.352 & -0.583 & 0.228 & -0.037 & 0.125 & 0.279 & 0.272 & -0.323 & 0.207 & 1 & & \\
\hline 2Y-STAR & 0.169 & 0.348 & -0.533 & 0.192 & -0.099 & 0.206 & 0.205 & 0.221 & -0.205 & 0.183 & 0.842 & 1 & \\
\hline ESC & 0.017 & -0.159 & 0.184 & -0.043 & 0.13 & -0.207 & 0.003 & -0.034 & -0.064 & -0.053 & -0.183 & -0.684 & 1 \\
\hline
\end{tabular}


Table 4

14 RAM and Harmonic Mean (HM) of the Ranks for 58 Leading Finance Journals

\begin{tabular}{|c|c|c|c|c|c|c|c|c|c|c|c|c|c|c|c|c|}
\hline Journal & HM & 2YIF & 2 YIF* $^{*}$ & IFI & 5YIF & $\begin{array}{c}\text { Imme- } \\
\text { diacy }\end{array}$ & 5YD2 & $\begin{array}{c}\mathrm{h}- \\
\text { index }\end{array}$ & СЗРО & $\begin{array}{c}\text { PI- } \\
\text { BETA }\end{array}$ & $\begin{array}{l}\text { Eigen- } \\
\text { factor }\end{array}$ & AI & CAI & ICQ & ESC & $\begin{array}{r}\text { 2YIF- } \\
\text { HM }\end{array}$ \\
\hline J FINANC & 1 & 1 & 1 & 15 & 1 & 1 & 20 & 1 & 4 & 35 & 3 & 1 & 3 & 4 & 19 & 0 \\
\hline J FINANC ECON & 2 & 3 & 3 & 24 & 3 & 2 & 17 & 2 & 1 & 1 & 2 & 3 & 2 & 6 & 31 & 1 \\
\hline REV FINANC STUD & 3 & 4 & 4 & 26 & 2 & 4 & 11 & 4 & 3 & 4 & 1 & 2 & 1 & 5 & 35 & 1 \\
\hline J ACCOUNT ECON & 4 & 2 & 2 & 36 & 4 & 10 & 48 & 5 & 2 & 2 & 9 & 8 & 6 & 31 & 35 & -2 \\
\hline ABACUS & 5 & 36 & 25 & 1 & 37 & 42 & 35 & 43 & 46 & 46 & 52 & 50 & 50 & 53 & 1 & 31 \\
\hline J MONETARY ECON & 6 & 12 & 11 & 19 & 10 & 26 & 16 & 3 & 5 & 10 & 4 & 5 & 4 & 3 & 38 & 6 \\
\hline IMF STAFF PAPERS & 7 & 7 & 5 & 1 & 31 & 55 & 57 & 29 & 21 & 8 & 28 & 19 & 16 & 13 & 12 & 0 \\
\hline GENEVA RISK INS REV & 8 & 42 & 33 & 1 & 48 & 46 & 49 & 55 & 45 & 26 & 55 & 44 & 41 & 33 & 2 & 34 \\
\hline IMF ECON REV & 9 & 5 & 6 & 28 & 9 & 55 & 50 & 43 & 40 & 41 & 27 & 4 & 5 & 2 & 7 & -4 \\
\hline ANNU REV FINANC ECON & 10 & 44 & 34 & 10 & 50 & 42 & 55 & 51 & 47 & 42 & 43 & 21 & 28 & 1 & 7 & 34 \\
\hline WORLD BANK ECON REV & 11 & 18 & 14 & 6 & 8 & 30 & 3 & 14 & 7 & 22 & 17 & 10 & 10 & 21 & 12 & 7 \\
\hline SIAM J FINANC MATH & 12 & 38 & 28 & 1 & 42 & 55 & 52 & 51 & 51 & 39 & 43 & 32 & 31 & 17 & 12 & 26 \\
\hline J ACCOUNT RES & 13 & 9 & 8 & 33 & 5 & 12 & 15 & 6 & 8 & 14 & 10 & 9 & 9 & 26 & 41 & -4 \\
\hline FORBES & 14 & 56 & 51 & 1 & 58 & 32 & 58 & 57 & 58 & 58 & 26 & 56 & 58 & 31 & 12 & 42 \\
\hline J FINANC QUANT ANAL & 15 & 13 & 10 & 8 & 13 & 17 & 31 & 8 & 12 & 16 & 7 & 7 & 8 & 7 & 22 & -2 \\
\hline EUR ACCOUNT REV & 16 & 45 & 43 & 32 & 25 & 55 & 1 & 40 & 41 & 45 & 42 & 42 & 45 & 49 & 35 & 29 \\
\hline ACCOUNT REV & 17 & 6 & 9 & 39 & 6 & 3 & 25 & 7 & 29 & 56 & 8 & 18 & 27 & 45 & 43 & -11 \\
\hline J FINANC INTERMED & 18 & 8 & 7 & 8 & 11 & 41 & 43 & 19 & 13 & 15 & 12 & 6 & 7 & 11 & 12 & -10 \\
\hline ACCOUNT ORG SOC & 19 & 10 & 12 & 37 & 7 & 34 & 9 & 12 & 9 & 3 & 20 & 25 & 19 & 48 & 41 & -9 \\
\hline J RISK UNCERTAINTY & 20 & 11 & 15 & 42 & 14 & 25 & 40 & 15 & 6 & 5 & 21 & 16 & 13 & 19 & 48 & -9 \\
\hline
\end{tabular}


Table 5

10 RAM and Harmonic Mean (HM) of the Ranks for 88 Leading Finance Journals

\begin{tabular}{|c|c|c|c|c|c|c|c|c|c|c|c|c|}
\hline Journal & $\mathbf{H M}$ & 2YIF & 2YIF* & 5YIF & Immediacy & 5YD2 & h-index & CЗPO & PI-BETA & Eigenfactor & ESC & 2YIF-HM \\
\hline J FINANC & 1 & 1 & 1 & 1 & 1 & 42 & 1 & 4 & 39 & 3 & 30 & 0 \\
\hline J FINANC ECON & 2 & 3 & 3 & 3 & 2 & 38 & 2 & 1 & 1 & 2 & 45 & 1 \\
\hline REV FINANC STUD & 3 & 4 & 4 & 2 & 6 & 29 & 4 & 3 & 4 & 1 & 50 & 1 \\
\hline J ACCOUNT ECON & 4 & 2 & 2 & 5 & 16 & 77 & 5 & 2 & 2 & 9 & 50 & -2 \\
\hline ACCOUNT AUDIT ACCOUN & 5 & 40 & 41 & 4 & 8 & 2 & 61 & 67 & 70 & 44 & 4 & 35 \\
\hline J MONETARY ECON & 6 & 12 & 11 & 12 & 35 & 36 & 3 & 5 & 10 & 4 & 54 & 6 \\
\hline J RISK MODEL VALIDAT & 7 & 88 & 87 & 87 & 76 & 1 & 84 & 83 & 82 & 84 & 16 & 81 \\
\hline INT INSOLV REV & 8 & 85 & 83 & 84 & 76 & 23 & 87 & 86 & 85 & 86 & 1 & 77 \\
\hline ACCOUNT REV & 9 & 6 & 9 & 7 & 4 & 48 & 7 & 29 & 76 & 8 & 62 & -3 \\
\hline J ACCOUNT RES & 10 & 9 & 8 & 6 & 18 & 35 & 6 & 8 & 14 & 10 & 60 & -1 \\
\hline ACCOUNT ORG SOC & 11 & 10 & 12 & 8 & 46 & 26 & 12 & 9 & 3 & 22 & 60 & -1 \\
\hline J RISK UNCERTAINTY & 12 & 11 & 16 & 18 & 34 & 68 & 15 & 6 & 5 & 23 & 70 & -1 \\
\hline J FINANC QUANT ANAL & 13 & 13 & 10 & 16 & 25 & 57 & 8 & 12 & 16 & 7 & 35 & 0 \\
\hline J BANK FINANC & 14 & 23 & 30 & 21 & 26 & 55 & 9 & 17 & 6 & 5 & 67 & 9 \\
\hline J FINANC INTERMED & 15 & 8 & 7 & 13 & 55 & 71 & 19 & 13 & 15 & 12 & 16 & -7 \\
\hline WORLD BANK ECON REV & 16 & 21 & 15 & 9 & 39 & 12 & 14 & 7 & 22 & 19 & 16 & 5 \\
\hline IMF STAFF PAPERS & 17 & 7 & 5 & 40 & 76 & 87 & 29 & 21 & 8 & 30 & 16 & -10 \\
\hline IMF ECON REV & 18 & 5 & 6 & 10 & 76 & 79 & 48 & 44 & 51 & 29 & 11 & -13 \\
\hline ABACUS & 19 & 46 & 29 & 52 & 56 & 63 & 48 & 54 & 57 & 68 & 2 & 27 \\
\hline MATH FINANC & 20 & 35 & 23 & 35 & 10 & 40 & 18 & 10 & 6 & 20 & 35 & 15 \\
\hline
\end{tabular}


Table 6

Correlations of 14 RAM and Harmonic Mean (HM) of the Ranks for 58 Leading Finance Journals

\begin{tabular}{|c|c|c|c|c|c|c|c|c|c|c|c|c|c|c|c|}
\hline Journal & 2YIF & 2YIF* & IFI & 5YIF & $\begin{array}{c}\text { Imme- } \\
\text { diacy }\end{array}$ & 5YD2 & h-index & СЗРО & PI-BETA & $\begin{array}{l}\text { Eigen- } \\
\text { factor }\end{array}$ & AI & CAI & ICQ & ESC & HM \\
\hline 2YIF & 1 & & & & & & & & & & & & & & \\
\hline 2YIF* & 0.894 & 1 & & & & & & & & & & & & & \\
\hline IFI & 0.115 & 0.11 & 1 & & & & & & & & & & & & \\
\hline 5YIF & 0.798 & 0.902 & 0.114 & 1 & & & & & & & & & & & \\
\hline Immediacy & 0.441 & 0.469 & -0.103 & 0.49 & 1 & & & & & & & & & & \\
\hline 5YD2 & -0.048 & 0.004 & -0.034 & 0.314 & 0.13 & 1 & & & & & & & & & \\
\hline h-index & 0.641 & 0.679 & 0.028 & 0.769 & 0.598 & 0.265 & 1 & & & & & & & & \\
\hline СЗРО & 0.703 & 0.737 & 0.178 & 0.819 & 0.573 & 0.293 & 0.907 & 1 & & & & & & & \\
\hline PI-BETA & 0.586 & 0.579 & 0.108 & 0.591 & 0.373 & 0.137 & 0.65 & 0.839 & 1 & & & & & & \\
\hline Eigenfactor & 0.632 & 0.705 & 0.247 & 0.82 & 0.57 & 0.222 & 0.864 & 0.837 & 0.563 & 1 & & & & & \\
\hline AI & 0.746 & 0.794 & 0.384 & 0.848 & 0.451 & 0.204 & 0.719 & 0.821 & 0.599 & 0.839 & 1 & & & & \\
\hline CAI & 0.765 & 0.796 & 0.357 & 0.848 & 0.461 & 0.207 & 0.745 & 0.879 & 0.723 & 0.825 & 0.979 & 1 & & & \\
\hline ICQ & 0.405 & 0.387 & 0.573 & 0.373 & 0.218 & -0.028 & 0.385 & 0.506 & 0.373 & 0.557 & 0.776 & 0.742 & 1 & & \\
\hline ESC & -0.042 & -0.103 & 0.731 & -0.067 & -0.312 & -0.062 & -0.216 & -0.061 & -0.057 & -0.014 & 0.144 & 0.132 & 0.257 & 1 & \\
\hline HM & 0.568 & 0.552 & 0.626 & 0.611 & 0.212 & 0.093 & 0.421 & 0.548 & 0.427 & 0.583 & 0.713 & 0.709 & 0.587 & 0.376 & 1 \\
\hline
\end{tabular}

Note: The Harmonic Mean of the ranks is given as HM. 
Table 7

Correlations of 11 RAM and Harmonic Mean (HM) of the Ranks for 88 Leading Finance Journals

\begin{tabular}{|c|c|c|c|c|c|c|c|c|c|c|c|}
\hline Journal & 2YIF & 2 YIF* & 5YIF & Immediacy & 5YD2 & h-index & C3PO & PI-BETA & Eigenfactor & ESC & HM \\
\hline 2YIF & 1 & & & & & & & & & & \\
\hline $2 Y I F *$ & 0.916 & 1 & & & & & & & & & \\
\hline $5 Y I F$ & 0.918 & 0.872 & 1 & & & & & & & & \\
\hline Immediacy & 0.594 & 0.489 & 0.549 & 1 & & & & & & & \\
\hline 5YD2 & -0.311 & -0.239 & 0.032 & -0.228 & 1 & & & & & & \\
\hline h-index & 0.779 & 0.777 & 0.743 & 0.537 & -0.196 & 1 & & & & & \\
\hline C3PO & 0.799 & 0.822 & 0.772 & 0.515 & -0.162 & 0.955 & 1 & & & & \\
\hline PI-BETA & 0.709 & 0.702 & 0.663 & 0.434 & -0.213 & 0.794 & 0.899 & 1 & & & \\
\hline Eigenfactor & 0.781 & 0.827 & 0.785 & 0.492 & -0.138 & 0.876 & 0.846 & 0.675 & 1 & & \\
\hline ESC & -0.168 & 0.115 & -0.11 & -0.309 & 0.118 & -0.178 & -0.087 & -0.103 & -0.018 & 1 & \\
\hline HM & 0.612 & 0.690 & 0.673 & 0.377 & 0.14 & 0.581 & 0.648 & 0.528 & 0.625 & 0.327 & 1 \\
\hline
\end{tabular}

Note: The Harmonic Mean of the ranks is given as HM. 


\section{References}

Bergstrom C. (2007), Eigenfactor: Measuring the value and prestige of scholarly journals, C\&RL News, 68, 314-316.

Bergstrom, C.T. and. J.D. West (2008), Assessing citations with the Eigenfactor ${ }^{\mathrm{TM}}$ metrics, Neurology, 71, 1850-1851.

Bergstrom, C.T., J.D. West and M.A. Wiseman (2008), The Eigenfactor ${ }^{\mathrm{TM}}$ metrics, Journal of Neuroscience, 28(45), 1143311434 (November 5, 2008).

Chang, C.-L. and M. McAleer (2012), Citations and impact of ISI tourism and hospitality journals, Tourism Management Perspectives, 1(1), 2-8.

Chang, C.-L. and M. McAleer (2013a), Ranking journal quality by harmonic mean of ranks: An application to ISI statistics \& probability, Statistica Neerlandica, 67(1), 27-53.

Chang, C.-L. and M. McAleer (2013b), What do experts know about forecasting journal quality? A comparison with ISI research impact in finance, Annals of Financial Economics, 8(1), 1-30.

Chang, C.-L. and M. McAleer (2013c), Ranking leading econometrics journals using citations data from ISI and RePEc, Econometrics, 1, 217-235.

Chang, C.-L. and M. McAleer (2014a), How should journal quality be ranked? An application to agricultural, energy, environmental and resource economics, Journal of Reviews on Global Economics, 3, 33-47.

Chang, C.-L. and M. McAleer (2014b), Quality weighted citations versus total citations in the sciences and social sciences, Tinbergen Institute Discussion Paper 14-023/III, Tinbergen Institute, The Netherlands. 
Chang, C.-L. and M. McAleer (2014c), Ranking economics and econometrics ISI journals by quality weighted citations, Review of Economics, 65(1), 35-52.

Chang, C.-L., E. Maasoumi and M. McAleer (2014), Robust ranking of journal quality: An application to economics, to appear in Econometric Reviews.

Chang, C.-L., M. McAleer and L. Oxley (2011a), What makes a great journal great in economics? The singer not the song, Journal of Economic Surveys, 25(2), 326-361.

Chang, C.-L., M. McAleer and L. Oxley (2011b), What makes a great journal great in the sciences? Which came first, the chicken or the egg?, Scientometrics, 87(1), 17-40.

Chang, C.-L., M. McAleer and L. Oxley (2011c), Great expectatrics: Great papers, great journals, great econometrics, Econometric Reviews, 30(6), 583-619.

Chang, C.-L., M. McAleer and L. Oxley (2011d), How are journal impact, prestige and article influence related? An application to neuroscience, Journal of Applied Statistics, 38(11), 2563-2573.

Chang, C.-L., M. McAleer and L. Oxley (2013), Coercive journal self citations, impact factor, journal influence and article influence, Mathematics and Computers in Simulation, 93, 190-197.

Hirsch, J.E. (2005), An index to quantify an individual's scientific research output, Proceedings of the National Academy of Sciences of the United States of America, 102(46), 16569-15572 (November 15, 2005).

ISI Web of Science (2013), Journal Citation Reports, Essential Science Indicators, Thomson Reuters ISI.

Wilhite, A.W. and E.A. Fong (2012), Coercive citation in academic publishing, Science, 335 (6068), 542-543. 